\title{
The Prefectural Participation Rates of Lung Cancer Screening Had a Negative Correlation with the Lung Cancer Mortality Rates
}

\author{
Motoyasu Sagawa ${ }^{1 *}$, Ryoko Machii ${ }^{2}$, Tomio Nakayama ${ }^{3}$, Takafumi Sugawara ${ }^{4}$, \\ Naoya Ishibashi ${ }^{4}$, Hideki Mitomo ${ }^{4}$, Takashi Kondo ${ }^{4}$, Toshiharu Tabata ${ }^{4}$
}

\begin{abstract}
Background: The participation rate is one of the most important indexes in the cancer screening. Historically in Japan, each local government has developed their own equations to calculate the subjects for population-based screening, which were different from each other, and therefore the participation rates of screening were not comparable. Recently, local governments were ordered to use the standardized equation in reporting data, which made it possible to compare the participation rates of cancer screening nationwide for the first time. We therefore investigated the correlation between the prefectural lung cancer mortality and several indicators of lung cancer screening. Methods: The prefectural participation rates of lung, gastric and colonic cancer screening, test positive rates, attendance rates for further examination, lung cancer detection rates and positive predictive values of lung cancer screening were collected from "Cancer Registration and Statistics" of the National Cancer Research Center website. The age-adjusted lung, gastric and colonic cancer mortality rates, smoking rates were also collected. The EZR software program was used for statistical analyses. Results: The participation rates of lung cancer screening had a strong positive correlation with the participation rates of gastric/colonic cancer screening $(\mathrm{P}<0.001)$. The prefectural lung cancer mortality rates had a moderate to weak negative correlation with the participation rates of lung cancer screening $(\mathrm{P}=0.009)$. A little correlation was noted between other quality assurance indicators of lung cancer screening and lung cancer mortality rates. Conclusion: These results suggested that participating in lung cancer screening might help reduce lung cancer mortality rates in some extent.
\end{abstract}

Keywords: lung cancer- cancer screening- mortality- ecological study- correlation

Asian Pac J Cancer Prev, 20 (3), 855-861

\section{Introduction}

Lung cancer is the leading cause of cancer deaths in many countries including Japan (Ferlay et al., 2013; Mathew et al., 2017; Nakagawa-Senda et al., 2017). In order to reduce cancer mortality by screening, it may be necessary to increase the participation rate of screening as well as to conduct effective screening tests with appropriate quality control. Several investigators were reported the relationship between the participation rate of cervical, breast and colorectal cancer screening and the mortality (Cramer, 1974; Laara et al., 1987; LazcanoPonce et al., 2008; Harding et al., 2015, Diniz CSG et al., 2017; Lynge et al., 2017; Yoshida et al., 2018). However, no reports in the English literature have explored the relationship between the participation rate of lung cancer screening and lung cancer mortality. In Japan, six case-control studies were conducted independently (Sobue et al., 1992; Okamoto et al., 1999; Nishii et al., 2001; Sagawa et al., 2001; Tsukada et al., 2001; Nakayama et al., 2002). The odds ratios of these studies for undergoing the screening within 12 months before diagnosis with regard to death from lung cancer were in the range of 0.40 to 0.72 , and significant mortality reduction was observed in 4 of these 6 studies. Based on the results, lung cancer screening has been conducted nationwide in Japan, although most western countries have not adopted. Therefore, the relationship between the participation rate of lung cancer screening and mortality should be reported from Japan.

In Japan, two lung cancer screening systems are employed nationwide (Nakatsuka et al., 1991). One is workplace-based screening, wherein lung cancer and pulmonary tuberculosis screening by chest roentgenogram is performed annually under the Industrial Safety and

${ }^{1}$ Division of Endoscopy, Faculty of Medicine, ${ }^{4}$ Division of Chest Surgery, Faculty of Medicine, Tohoku Medical and Pharmaceutical University, Sendai, ${ }^{2}$ Division of Medical Support and Partnership, Center for Cancer Control and Information Service, ${ }^{3}$ Division of Screening Assessment and Management, Center for Public Health Service, National Cancer Center, Tokyo, Japan. *For Correspondence: sagawam@tohoku-mpu.ac.jp 
Health Act under the responsibility of the employer. The subjects targeted by this workplace-based screening approach are employees of companies. The other system is population-based cancer screening, wherein lung cancer and pulmonary tuberculosis screening by chest roentgenogram (additional sputum cytology for heavy smokers) is performed annually under the Health Promotion Act (former Health and Medical Service Law for the Aged) under the responsibility of the local municipality government. The subjects targeted by this population-based screening are residents in the municipality other than those receiving workplace-based screening.

However, for small companies, the employers are permitted to unprovide workplace-based screening for their employees, so the employees of such companies become additional target subjects for population-based screening, which is complicated system and causes some confusion. In this situation, local governments have imprecise data on the subjects targeted for populationbased screening. Given this confusion and the fact that the Ministry of Health, Labour and Welfare has ordered local governments to submit the number of subjects for population-based screening to nationwide reporting system, local governments have developed their own unique equations for calculating the subjects that should be targeted for population-based screening. However, these equations necessarily differ among municipalities, so the participation rates of the screening in the different municipalities have not been comparable, which is one of the most important reasons why it has not been reported the relationship between the participation rate of lung cancer screening and mortality from Japan.

To resolve various issues associated with the quality assurance of population-based cancer screening, Saito's Team was organized by the Ministry of Health, Labour and Welfare. Saito's Team addressed the issue of incomparable participation rates, and developed the standardized equation for calculating the number of targeted subjects, as described below.

Standardized number of targeted subjects for population-based cancer screening $=$ the number of population - (the number of employed workers - the number of engaged workers in agriculture, forestry and fisheries)

From 2010, local governments were ordered to use the standardized equation in reporting data to the Ministry of Health, Labour and Welfare. This made it possible to compare the participation rates among different local governments for the first time. Therefore, we herein report the correlation among prefectural lung cancer mortality and several indexes concerning cancer screening.

\section{Materials and Methods}

In 47 prefectures of Japan, 37,121,822 people were the estimated targeted subjects for population-based lung cancer screening in 2012, and 18,698,345 people of them were 40-69 years of age. The correlations among several prefectural indexes about cancer screening were investigated.
The following data were downloaded from the Cancer Information Service "Cancer Registration and Statistics" of the National Cancer Research Center website (https:// ganjoho.jp/reg_stat/statistics/index.html, download date: October 1, 2017). The original data were published in the Report on Regional Public Health Services and Health Promotion Services, Report of Vital Statistics, and Comprehensive Survey of Living Conditions. The participation rates of cancer screening in the Report on Regional Public Health Services and Health Promotion Services were calculated with the standardized equation during the examined period.

1) Prefectural participation rate of lung, gastric and colonic cancer screening (2010-2012, 40-69 years of age).

2) Prefectural test positive rate, attendance rate for further examination, lung cancer detection rate, and positive predictive value of lung cancer screening (2011-2013, 40-74 years of age).

3) Prefectural age-adjusted lung, gastric and colonic cancer mortality rate (2013-2015, $\leq 74$ years of age).

4) Prefectural smoking rate (2013, $\geq 20$ years of age).

In general, the average values for the most recent three years in available data were used. However, since the smoking rates were obtained from the "Comprehensive Survey of Living Conditions", which is conducted only once every three years, the latest value was used for smoking rates. In addition, since the proportion of very elderly people greatly influenced many indexes, such as the mortality and participation rate, data with an upper age limit were used if available.

Based on the above data, we analyzed the correlation for the following indicators by each prefecture:

1) Correlation between the participation rates of lung cancer screening and gastric cancer screening.

2) Correlation between the participation rates of lung cancer screening and colonic cancer screening.

3) Correlation between the participation rates of lung cancer screening and the attendance rates for further examinations.

4) Correlation between the participation rates of lung cancer screening and the smoking rates.

5) Correlation between the participation rates of lung cancer screening and the lung cancer mortality rates.

6) Correlation between the participation rates of lung cancer screening and the gastric cancer mortality rates.

7) Correlation between the participation rates of lung cancer screening and the colonic cancer mortality rates.

8) Correlation between the lung cancer mortality rates and the smoking rates.

9) Correlation between the lung cancer mortality rates and the test positive rates of lung cancer screening.

10) Correlation between the lung cancer mortality rates and the attendance rates for further examinations.

11) Correlation between the lung cancer mortality rates and the lung cancer detection rates of lung cancer screening.

12) Correlation between the lung cancer mortality rates and the positive predictive values of lung cancer screening.

The EZR software program (Kanda, 2013) was used for the statistical analyses, and the significance level was set at $5 \%$. 


\section{Results}

Correlation between the participation rates of lung cancer screening and gastric cancer screening (Supplemental Figure 1).

The participation rates of lung cancer screening and gastric cancer screening in both males and females had a strong positive correlation, with a correlation coefficient of $0.83-0.84$. The prefectures with high participation rates of lung cancer screening tended to have high participation rates of gastric cancer screening.

Correlation between the participation rates of
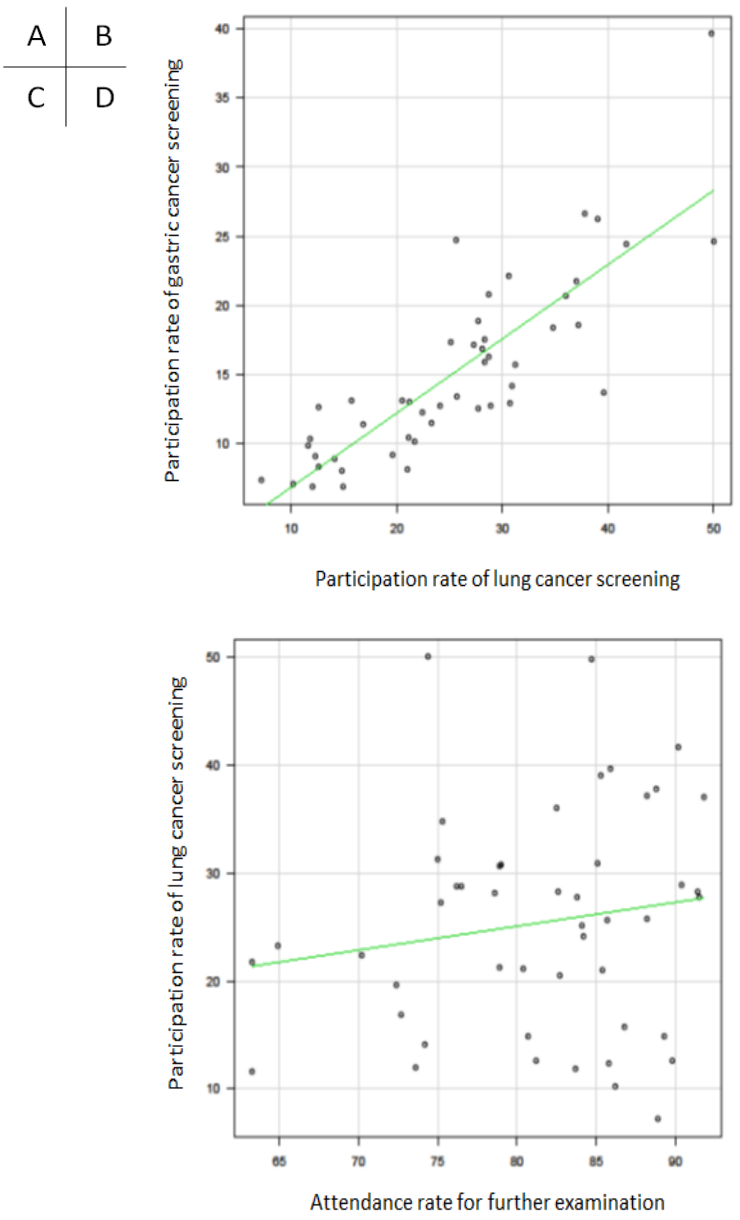

lung cancer screening and colonic cancer screening (Supplemental Figure 2).

The participation rates of lung cancer screening and colonic cancer screening in both males and females had a strong positive correlation, with a correlation coefficient of 0.82 . The prefectures with high participation rates of lung cancer screening tended to have high participation rates of gastric cancer screening.

Correlation between the participation rates of lung cancer screening and the attendance rates for further examinations (Supplemental Figure 3)

There was no correlation between the participation
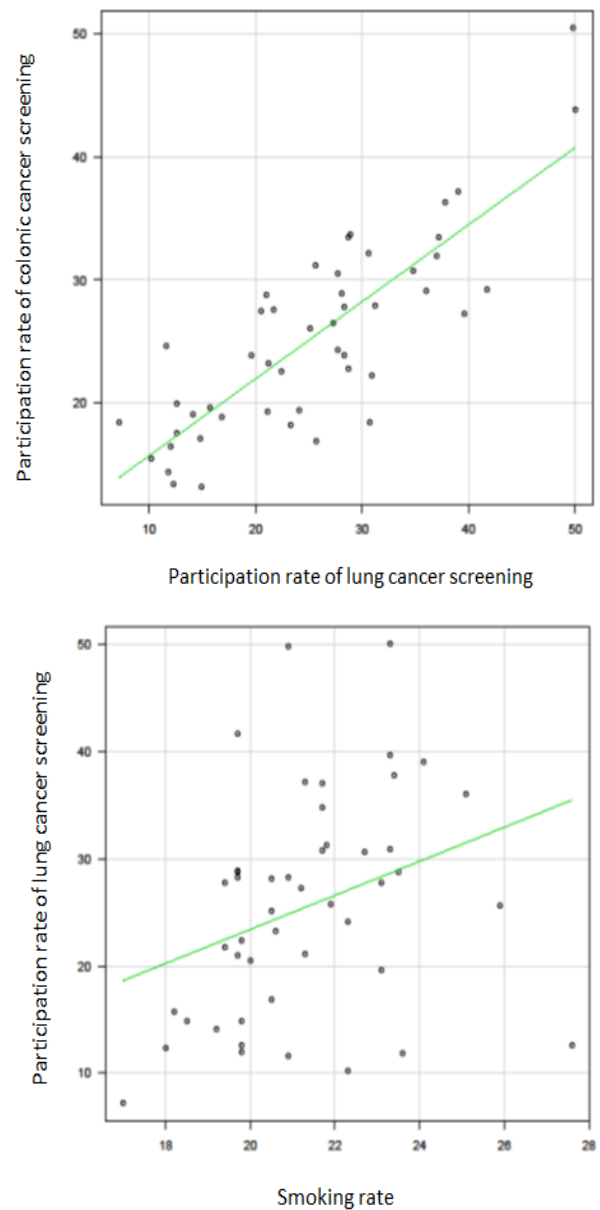

Figure 1. The Correlation (in Total: Males and Females) between the Participation Rates of Lung Cancer Screening and Other Factors. A, the Participation Rates of Gastric Cancer Screening; B, the Participation Rates of Colonic Cancer Screening; C, the Attendance Rates for Further Examination of Lung Cancer Screening; D, the Smoking Rates.

Table 1. The Correlations between the Prefectural Participation Rates of Lung Cancer Screening and Other Factors

\begin{tabular}{lcccccc}
\hline Other factor & \multicolumn{2}{c}{ Males } & \multicolumn{3}{c}{ Females } & \multicolumn{3}{c}{ Total } \\
& $\mathrm{CC}$ & $\mathrm{p}$ value & $\mathrm{CC}$ & $\mathrm{p}$ value & $\mathrm{CC}$ & $\mathrm{p}$ value \\
\hline The participation rates of gastric cancer screening & 0.84 & $<0.001$ & 0.83 & $<0.001$ & 0.84 & $<0.001$ \\
The participation rates of colonic cancer screening & 0.82 & $<0.001$ & 0.82 & $<0.001$ & 0.82 & $<0.001$ \\
The attendance rates for the further examination of lung cancer screening & 0.05 & 0.724 & 0.21 & 0.163 & 0.16 & 0.292 \\
The smoking rates & 0.48 & $<0.001$ & 0.01 & 0.968 & 0.33 & 0.026 \\
The lung cancer mortality rates & -0.35 & 0.016 & -0.43 & 0.002 & -0.38 & 0.009 \\
The gastric cancer mortality rates & 0.09 & 0.547 & 0.10 & 0.492 & 0.13 & 0.378 \\
The colonic cancer mortality rates & 0.01 & 0.951 & -0.12 & 0.434 & -0.01 & 0.935 \\
\hline
\end{tabular}

CC, correlation coefficient 

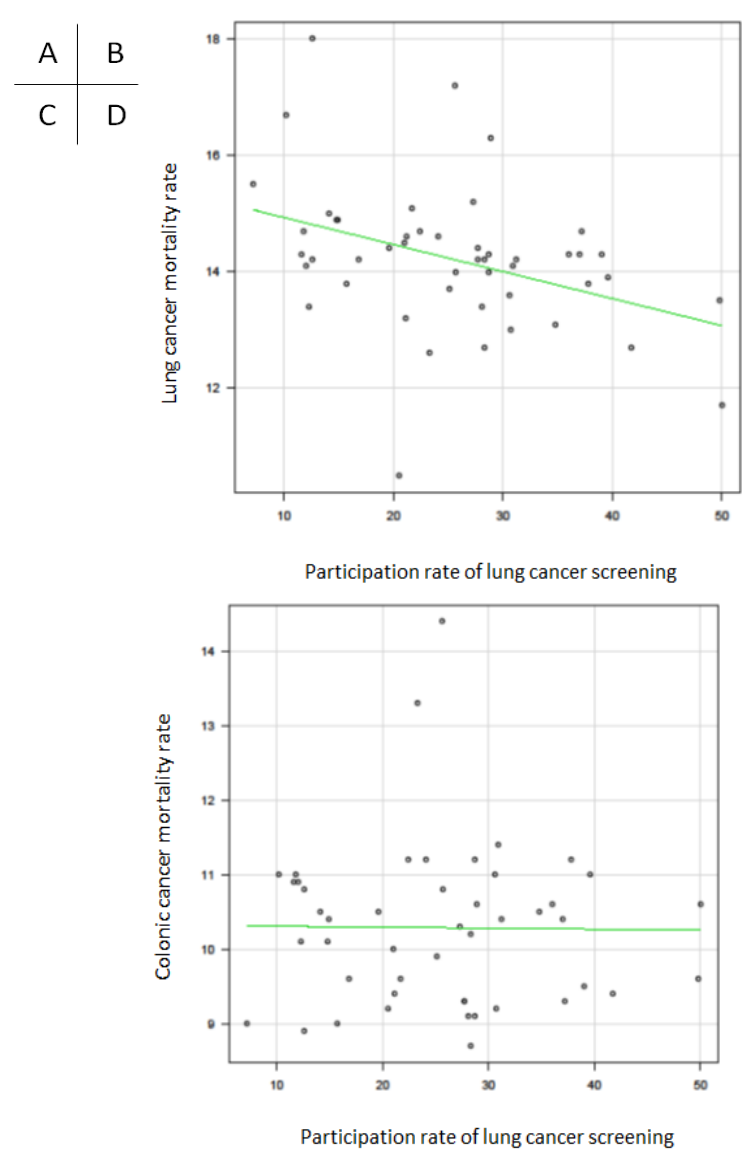
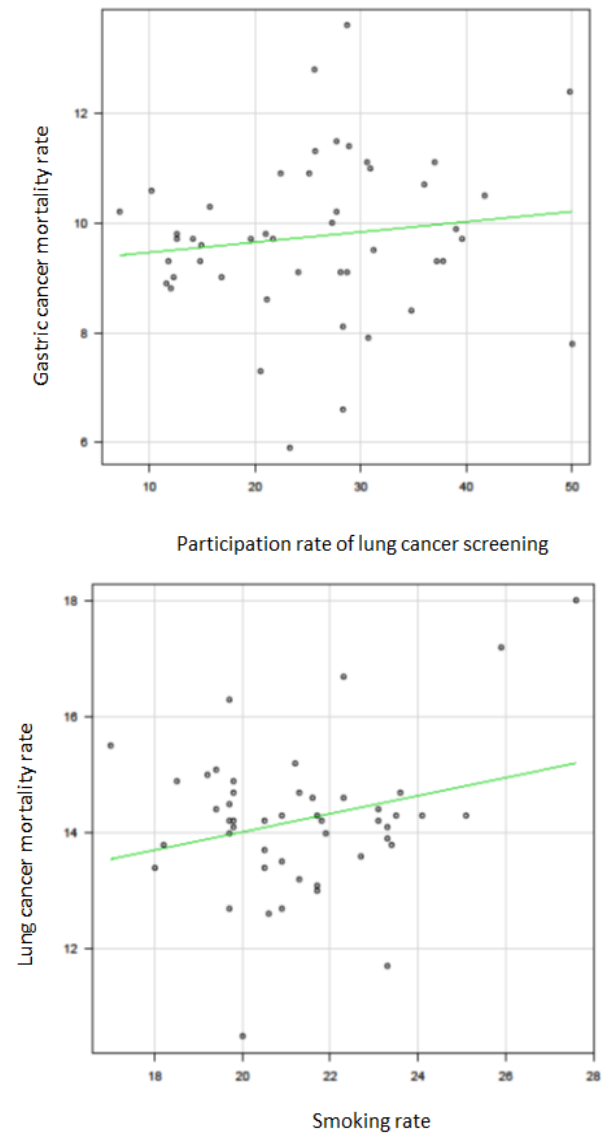

Figure 2. The Correlation between the Participation Rates of Lung Cancer Screening and the Lung Cancer Mortality Rates (A); the Gastric Cancer Mortality Rates (B); and the Colonic Cancer Mortality Rates (C); The Correlation between the Lung Cancer Mortality Rates and the Smoking Rates (D).

rates of lung cancer screening and attendance rates for further examination in males or females.

Correlation between the participation rates of lung cancer screening and the smoking rates (Supplemental Figure 4).

The participation rates of lung cancer screening and smoking rates in males had a positive correlation. However, there was no correlation in females.

Correlation between the participation rates of lung cancer screening and the lung cancer mortality rates (Supplemental Figure 5).

There was a negative correlation between the lung cancer mortality rates and the participation rates of lung cancer screening in both males and females. The prefectures with high participation rates of lung cancer screening tended to have lower lung cancer mortality rates.

Correlation between the participation rates of lung cancer screening and the gastric cancer mortality rates (Supplemental Figure 6).

There was no correlation between the participation rates of lung cancer screening and the gastric cancer mortality rates in males or females.

Correlation between the participation rates of lung cancer screening and the colonic cancer mortality rates (Supplemental Figure7).

There was no correlation between the participation rates of lung cancer screening and the colonic cancer mortality rates in males or females.

Table 2. The Correlations between the Prefectural Lung Cancer Mortality Rates and Other Factors

\begin{tabular}{lcccccc}
\hline Other factor & \multicolumn{2}{c}{ Males } & \multicolumn{2}{c}{ Females } & \multicolumn{2}{c}{ Total } \\
& CC & $\mathrm{p}$ value & $\mathrm{CC}$ & $\mathrm{p}$ value & $\mathrm{CC}$ & $\mathrm{p}$ value \\
\hline The smoking rates & 0.14 & 0.345 & 0.57 & $<0.001$ & 0.26 & 0.078 \\
The test positive rates of lung cancer screening & -0.05 & 0.723 & -0.08 & 0.581 & -0.02 & 0.898 \\
The attendance rates for the further examination of lung cancer screening & 0.22 & 0.147 & -0.11 & 0.477 & 0.13 & 0.396 \\
The detection rates of lung cancer screening & 0.21 & 0.159 & 0.12 & 0.428 & 0.29 & 0.046 \\
The positive predictive values of lung cancer screening & 0.12 & 0.427 & 0.18 & 0.232 & 0.17 & 0.247 \\
\hline
\end{tabular}

$\mathrm{CC}$, correlation coefficient 

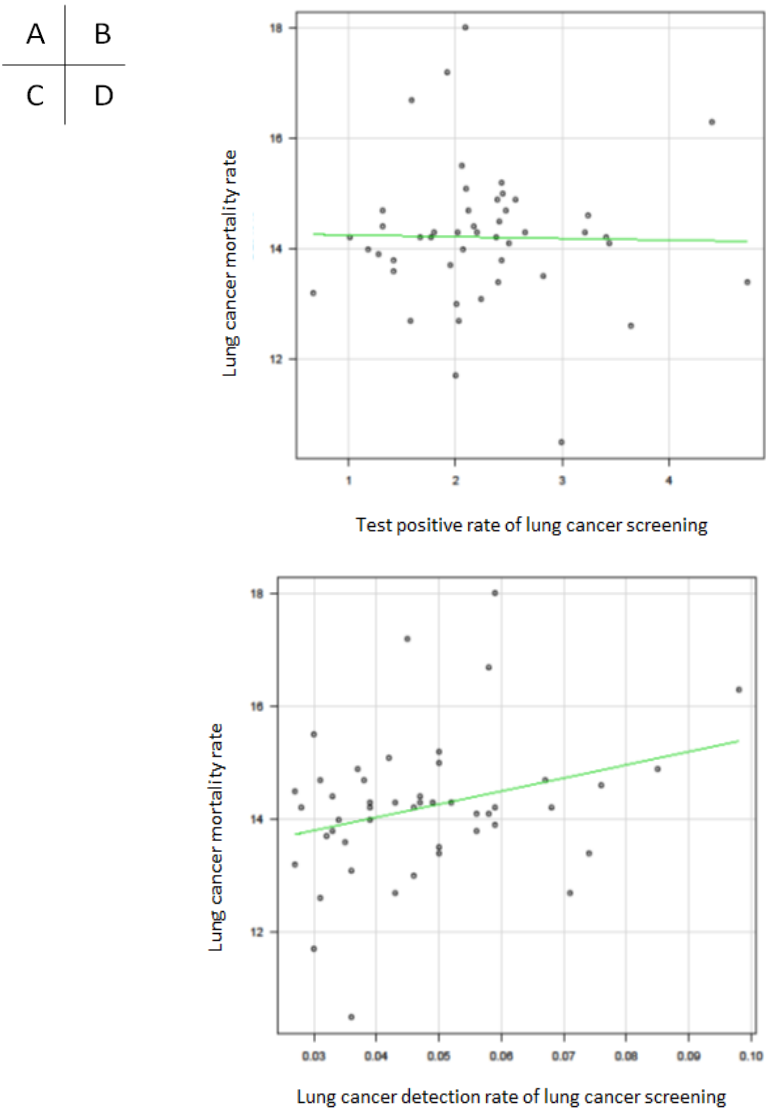
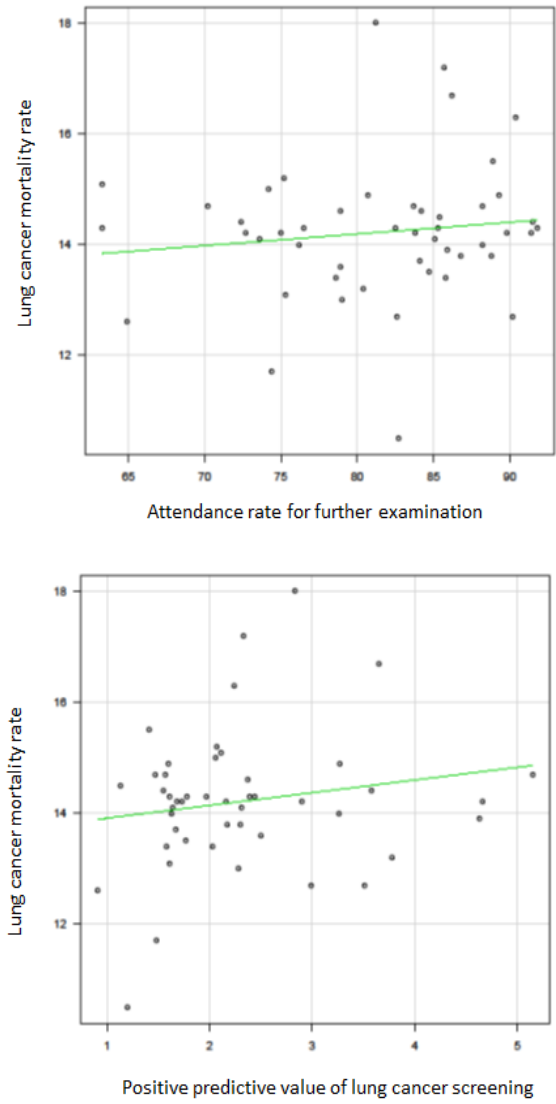

Figure 3. The Correlation between the Lung Cancer Mortality Rates and the Test Positive Rates (A); the attendance rates for further examination (B); lung cancer detection rates $(\mathrm{C})$; and the positive predictive values of lung cancer screening (D).

Correlation between the lung cancer mortality rates and the smoking rates (Supplemental Figure 8).

There was a positive correlation between the lung cancer mortality rates and the smoking rates in females, but no correlation was noted in males. The prefectures with high smoking rates tended to have higher lung cancer mortality rates only in females.

Correlation between the lung cancer mortality rates and the test positive rates of lung cancer screening (Supplemental Figure 9).

There was no correlation between the lung cancer mortality rates and the test positive rates of lung cancer screening in males or females.

Correlation between the lung cancer mortality rates and the attendance rates for further examinations (Supplemental Figure 10).

There was no correlation between the lung cancer mortality rates and the attendance rates for further examination in males or females.

Correlation between the lung cancer mortality rate and the lung cancer detection rate of lung cancer screening (Supplemental Figure 11).

Although there was no correlation between the lung cancer mortality rate and the lung cancer detection rate of lung cancer screening in males or females, there was a positive correlation in total (males and females).
Correlation between the lung cancer mortality rates and the positive predictive values of lung cancer screening (Supplemental Figure 12).

There was no correlation between the lung cancer mortality rates and the positive predictive values of lung cancer screening in males or females.

\section{Discussion}

The participation rate is one of the most important indexes in the cancer screening system. When a cancer screening modality has an effect on reducing mortality, the extent of the mortality reduction due to such cancer in a group depends on the screening participation rate. On the other hand, when a screening modality has no effect on reducing mortality, the participation rate naturally does not influence the mortality in the group. Therefore, evaluating the relationship between the cancer mortality and the participation rate of cancer screening is very important. Although several investigators have reported a correlation between the participation rate of other cancer screening and the respective cancer mortality (Cramer, 1974; Lazcano-Ponce et al., 2008; Harding et al., 2015, Diniz et al., 2017; Lynge et al., 2017; Yoshida et al., 2018), no reports have explored the relationship between the participation rate of lung cancer screening and the lung cancer mortality.

Historically in Japan, each local government has developed their own equation to calculate the number of 
the subjects for population-based screening, which were different from each other, so the participation rates of screening were not comparable. Recently, the standardized equation was developed, which made it possible to compare the participation rates of cancer screening nationwide for the first time. We therefore investigated the correlation between the lung cancer mortality and several indicators of lung cancer screening including the participation rates.

The results of this study revealed that the participation rates of lung cancer screening had a strong positive correlation with the participation rates of gastric/colonic cancer screening, suggesting that those who received some cancer screening also tended to receive other cancer screenings. However, this did not mean that they had "healthy habits", as the participation rates of lung cancer screening did not correlate with the smoking rates in females, and the high participation rates of lung cancer screening in males were conversely associated with the high smoking rates.

Lung cancer mortality rates had a moderate to weak negative correlation with the participation rates of lung cancer screening, which indicated that the prefectures with high participation rates of lung cancer screening tended to have low lung cancer mortality rates. This seemed to suggest that lung cancer screening may be helpful in reducing the lung cancer mortality rate in some extent, but we need to discuss three other possible reasons for the observed correlation.

First, the smoking rates of the subjects who participated in the lung cancer screening might have been low, which may have resulted in their lung cancer mortality rate being low as well. However, as mentioned above, the prefectures with high participation rates of lung cancer screening showed no correlation with the smoking rates in females. Furthermore, in males, high participation rates correlated with high smoking rates, so the hypothesis described above was rejected.

Second, the participation rates of lung cancer screening may have been related to healthy habits other than smoking (self-selection biases) or to some prefectural cancer control policies, which might have influenced the mortality rates. However, if this was the case, such healthy habits/cancer control policies would also influence other cancer mortality rates, and yet the participation rates of lung cancer screening were not associated with gastric or colonic cancer mortality rates. This indicates that the influence of healthy habits or prefectural cancer control policies was limited.

Third, the prefectures with lower lung cancer mortality rates may simply have tended to have a lower prevalence of lung cancer than other prefectures. Since no nationwide cancer registry has yet been established in Japan, the precise prefectural prevalence has not been reported. Although we cannot assess whether this hypothesis is true or not, we can think of no reason the prefectures with higher participation rates of lung cancer screening might have had a lower prevalence of lung cancer.

The observed moderate to weak negative correlation between the lung cancer mortality rates and the participation rates of lung cancer screening could not be attributed to any of the three possible reasons suggested, which might suggest that participating in lung cancer screening would help reduce lung cancer mortality rates in some extent. However, the correlation was not strong and there were possible other confounding factors, it should be cautious to conclude.

Other analyses showed a positive correlation between the smoking rates and the lung cancer mortality rates in females but no correlation in males. It is well-known that smoking is a major risk factor for lung cancer morbidity and mortality, so the reason why a positive correlation was not noted in males is unclear. One possible reason is that there was a positive correlation in males between the smoking rates and the participation rates of lung cancer screening, which had a negative correlation with lung cancer mortality rates; as such, these two factors may therefore have canceled each other out.

We also analyzed the correlation between the lung cancer mortality rates and the lung cancer detection rates on lung cancer screening. A weak positive correlation was observed in total (males and females), which might suggest that the lung cancer mortality rates were also high in areas with high lung cancer incidence rates, but we were unable to verify this, as the precise prefectural prevalence was not reported in Japan. We also examined the correlation between the lung cancer mortality rates and other quality assurance indicators of lung cancer screening, such as the test positive rates, the attendance rates for further examinations, and the positive predictive values, but no correlation was noted. Although the reasons for the lack of correlation are unclear, the influence of quality assurance is clearly limited in situations where the participation rate itself is low (the participation rate across the whole of Japan was $21.2 \%$ ), so the effect would be evident only when the participation rate rises.

Several issues associated with the present study remain to be discussed. First, since these results show the correlation of the numerical values of the statistical indexes with no direct causal relationship analysis, we cannot deny the involvement of other factors. Therefore, even though a significant correlation was noted, the relevance is merely presumed. Second, the most recent statistical indicators were used for the analyses, but the reference years used for these indicators were slightly different. However, since this study was an analysis of the correlation by prefectures, it was only necessary to know the overall trend. The statistical indexes of prefectures do not fluctuate greatly over short periods, and we used the average values over several years, so the slight differences in the reference year seemed to have a negligible effect. Third, although the standardized equation enabled the comparison of the participation rates of cancer screening among different municipalities, these rates were still "estimated values". In 2016, the Investigating Commission on Cancer Screening decided that "population over 40 years of age", which was an "actual value", would be counted as "the number of subjects eligible for population-based screening" after 2017. We hope this new definition of the targeted subjects will aid in improving the quality assurance of population-based screening as well as workplace-based screening. 
In conclusions using the standardized equation enabled the comparison of the nationwide participation rates of cancer screening, so we examined the correlation between lung cancer mortality rates and several quality assurance indicators of lung cancer screening by prefecture. Our evaluation showed that the lung cancer mortality rates had a moderate to weak negative correlation with the participation rates of lung cancer screening, suggesting that participation in lung cancer screening might reduce the mortality in some extent. A little correlation was noted between lung cancer mortality rates and other quality assurance indicators of lung cancer screening, which might suggest that the influence of the quality of screening was limited, especially in cases where the participation rates of screening were low. Because the present study was an ecological one which might have several biases, further studies will be required for the detailed assessment of the lung cancer screening.

\section{Acknowledgements}

We are grateful to Dr. Hiroshi Saito, former director of the Division of Screening Assessment and Management, Center for Public Health Service, National Cancer Center, Japan.

This work was partially supported by a Grant-in-Aid for Scientific Research from the Ministry of Education, Culture, Sports, Science and Technology [grant number 17H04125]; the Ministry of Health, Labour and Welfare [grant number H30-Gantaisaku-Ippan-002]; the National Cancer Center Research and Development Fund [grant number 29-A-16]; and by the Practical Research for Innovative Cancer Control of the Japan Agency for Medical Research and Development, AMED [grant number 18ck0106408], Japan.

\section{References}

Cramer DW (1974). The role of cervical cytology in the declining morbidity and mortality of cervical cancer. Cancer, 34, 2018-27.

Diniz CSG, Pellini ACG, Ribeiro AG, et al (2017). Breast cancer mortality and associated factors in São Paulo State, Brazil: an ecological analysis. BMJ Open, 7, e016395.

Ferlay J, Steliarova-Foucher E, Lortet-Tieulent J, et al (2013). Cancer incidence and mortality patterns in Europe: estimates for 40 countries in 2012. Eur J Cancer, 49, 1374-403.

Harding C, Pompei F, Burmistrov D, et al (2015). Breast cancer screening, incidence, and mortality across US counties. JAMA Intern Med, 175, 1483-9.

Kanda Y (2013). Investigation of the freely available easy-to-use software 'EZR' for medical statistics. Bone Marrow Transplant, 48, 452-8.

Laara E, Day NE, Hakama M (1987). Trends in mortality from cervical cancer in the Nordic countries: association with organised screening programmes. Lancet, 1, 1247-9.

Lazcano-Ponce E, Palacio-Mejia LS, Allen-Leigh B, et al (2008). Decreasing cervical cancer mortality in Mexico: effect of Papanicolaou coverage, birthrate, and the importance of diagnostic validity of cytology. Cancer Epidemiol Biomarkers Prev, 17, 2808-17.

Lynge E, Bak M, von Euler-Chelpin M, et al (2017). Outcome of breast cancer screening in Denmark. BMC Cancer, 17, 897.
Mathew A, Sara George P, Kalavathy MC, et al (2017). Cancer incidence and mortality: District cancer registry, trivandrum, South India. Asian Pac J Cancer Prev, 18, 1485-91.

Nakagawa-Senda H, Yamaguchi M, Matsuda T, et al (2017). Cancer prevalence in Aichi, Japan for 2012: Estimates based on incidence and survival data from population-based cancer registry. Asian Pac J Cancer Prev, 18, 2151-6.

Nakatsuka H, Watanabe T, Hisamichi S, et al (1991). Different rates of acceptance of health examination among the people under 2 separate health insurance programs in Japan. Tohoku J Exp Med, 163, 59-71.

Nakayama T, Baba T, Suzuki T, Sagawa M, Kaneko M (2002). An evaluation of chest X-ray screening for lung cancer in Gunma Prefecture, Japan: a population-based case-control study. Eur J Cancer, 38, 1380-7.

Nishii K, Ueoka H, Kiura K, et al (2001). A case-control study of lung cancer screening in Okayama Prefecture, Japan. Lung Cancer, 34, 325-32.

Okamoto T, Suzuki T, Hasegawa H, et al (1999). Evaluation of a clinic-based screening program for lung cancer with a case-control design in Kanagawa, Japan. Lung Cancer, 25, 77-85.

Sagawa M, Tsubono Y, Saito Y, et al (2001). A case-control study for evaluating the efficacy of mass screening program for lung cancer in Miyagi Prefecture, Japan. Cancer, 92, 588-94.

Sobue T, Suzuki T, Naruke T, Japanese Lung-Cancer-Screening Research Group (1992). A case control study for evaluating lung cancer screening in Japan. Int J Cancer, 50, 230-7.

Tsukada H, Kurita Y, Yokoyama A, et al (2001). An evaluation of screening for lung cancer in Niigata Prefecture, Japan: A population-based case-control study. Brit J Cancer, 85, 1326-31.

Yoshida Y, Schmaltz CL, Jackson-Thompson J, Simoes EJ (2018). The impact of screening on cancer incidence and mortality in Missouri, USA, 2004-2013. Public Health, 154, 51-8.

\section{(ब) $\mathbb{Q} \otimes$}

This work is licensed under a Creative Commons AttributionNon Commercial 4.0 International License. 